\title{
VirtES (Virtual Enterprise Simulator): A Proposed Methodology for Enterprise Simulation Modeling
}

\author{
Giovanni Davoli , Sergio A. Gallo, and Riccardo Melloni \\ Department of Mechanical and Civil Engineering (DIMeC) \\ University of Modena and Reggio Emilia \\ via Vignolese 905, 41100, Modena, Italy \\ giovanni.davoli@unimore.it
}

\begin{abstract}
In this paper a methodology to develop simulation models is presented. The methodology is based on a multi-level simulation model which allows flexibility and process analysis. The present work starts from applied researches in different SME enterprises. Enterprise management often needs easy and fast developed tools to increase production capacity and flexibility. In many cases performances increase is possible only adopting a BPR (business processes reengineering) approach. Nevertheless the resistance to a BPR approach is underlined in recent bibliography. The proposed approach consists of a three stages methodology, named VirtES (Virtual Enterprise Simulator). VirtES methodology was first applied to ceramic tiles enterprises. The results achieved encourage the adoption to other industrial field.
\end{abstract}

Keywords: BPR, process, simulation, tile, SME.

\section{Introduction}

The economic scenario today is highly competitive in terms of number of competitors and costs. To remain competitive, companies have to maintain a high-level of performance by maintaining high quality, low cost, low manufacturing lead times, and high customer satisfaction [1].

Enterprise management often needs easy and fast developed tools to increase production capacity and flexibility, even in a SME (Small Medium Enterprises) contest. Usually SMEs are organized by function and for achieving the expected results it is not enough to improve the performance of a single function. In many cases performances increase is possible only adopting a BPR (Business Processes Reengineering) approach. Nevertheless this approach meets mostly two objections from management. First of all the resources and the time involved represent an important investment, secondly it is not possible to exactly quantify the predictable results. The resistance to the most known BPR methodology is underlined in recent bibliography [2].

It could be argued that the development of simulation models is an useful approach to quantify the expected results before adopting any BPR action. In fact, because of its great versatility, flexibility, and power, simulation is one of the most widely used operations research techniques from the early eighties [3]. However several studies 
show that there is a low usage of simulation by industries [4], especially simulation has not been widely applied to SMEs (Small Medium Enterprises) [5].

To support BPR activities, a model of the whole enterprise is needed. Carry on the development of such a simulation model is a very expensive activity, in term of resources and time consumption, adopting a commercial simulation tool (for example: AutoMod $^{\mathrm{TM}}$, Arena ${ }^{\mathrm{TM}}$, em-Plant ${ }^{\mathrm{TM}}$ ). Moreover the developed model for a specific enterprise is not adaptable to another enterprise, because the model has to be very detailed. For this reason SMEs are prevented to develop simulation model in order to support BPR activities.

\section{The Proposed Methodology}

The proposed methodology was developed during several applied researches carried on in the last years. These researches were focused on the improvement of production - logistic systems performances in SMEs. To achieve the goal BPR activities were undertaken and simulation models were developed to support and drive the BPR process. The SMEs involved operate on different industrial fields such as: ceramic and tile, automotive, wood products and large distribution. Facing these activities a methodology was developed to answer the main common requests coming from different enterprises, this methodology is named VirtES (Virtual Enterprise Simulator).

\subsection{VirtES}

The aim is to provide an easy to use methodology to develop simulation model for supporting BPR in SMEs. A three stages methodology is developed to address the SMEs instances.

The first stage consists of developing a processes based model highly adaptable to manufactures operating in a specific field. The activities of business processes mining and business process analysis starts from one or more specific enterprises and then are enlarged to all company of the same industrial field. This analysis allowed to develop processes based model, for a generic enterprise, that consider the common features of all companies of the same industrial field. The processes model is developed according to the FDM (Factory Data Model) paradigm [6].

In the second stage a simulation model based on the processes model defined is developed. This is a "high level" simulation model that gives an overview of the performances of the enterprise in term of macro KPI (key performances indices). At this stage the behavior of the whole enterprise is simulated starting from order reception to products delivery. SciLab open source platform is adopted to develop the high level simulation model. Detailed simulation and optimization sub - models are provided at the third stage. These are "low level" simulation models, extremely detailed and focused on a specific sub system or process. The "low level" models can be properly developed with the most appropriate mathematical formalism or commercial simulation suite such as: AutoMod ${ }^{\mathrm{TM}}$, Arena ${ }^{\mathrm{TM}}$, em-Plant ${ }^{\mathrm{TM}}$. These models point out results in terms of production capacity, lead time, scheduling algorithm, exc. The three stages structure of VirtES methodology is shown in fig. 1. 


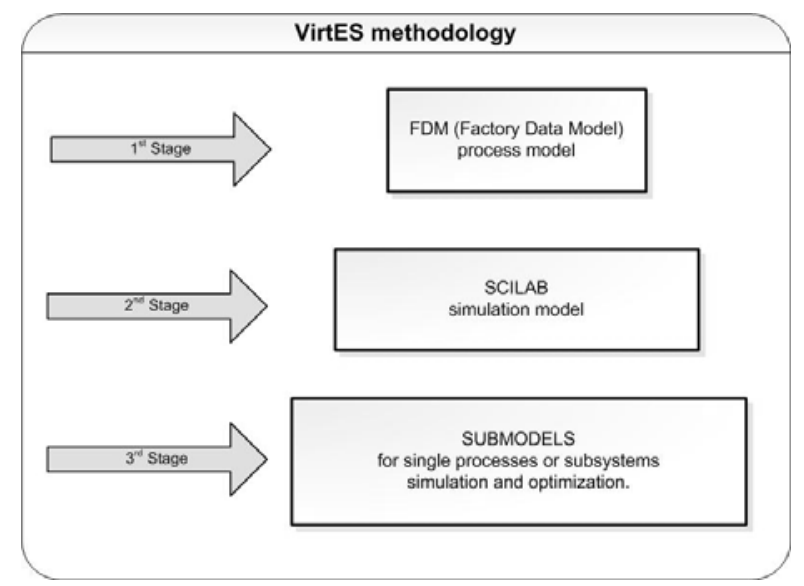

Fig. 1. There stages VirtES methodology structure

\subsection{The First Stage}

At the first stage, the aim is to develop a process model of the whole enterprise. At this stage a strong cooperation between the modeling team and the human resources of the enterprise is needed. Process modeling is an high resource and time consumption activity and this represent one of the main difficulties in SMEs.

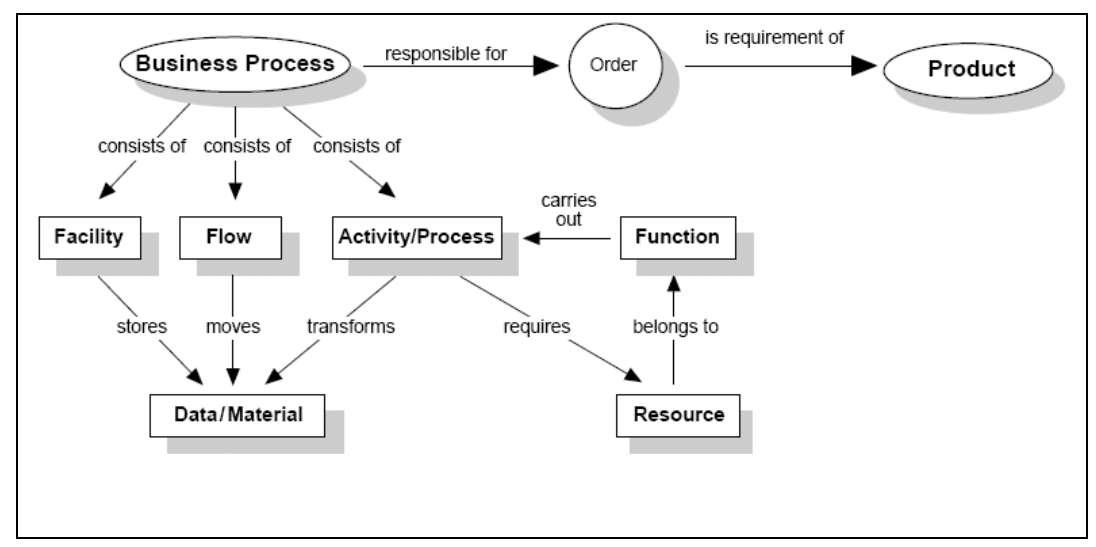

Fig. 2. FDM paradigm [6]

The FDM was introduced by $\mathrm{Yu}$ in the 2001 to accelerate the modeling process adopting a scalable and reusable model. The FDM model, belonging to the data model family, was chosen here mainly because of the flexibility of the model and the possibility of its use even when only partially complete. The FDM model paradigm is shown in fig. 2.

The development of the FDM model starts from the study of a specific enterprise. Then the model is compared with the model already available from previous studies and literature review, mainly with model of enterprises operating in the same 
industrial field. If a reference model is found this can be adapted to suite any particular features of the studied enterprise. Otherwise a new reference model is developed. The reference model has to collect the main characteristics of the studied enterprise and has to be simple to preserve the reusability.

\subsection{The Second Stage}

At the second stage the FDM process model is implemented on the open - source platform SciLab. An open - source platform was chosen to allow the integration with detailed sub-models developed with specific software at the third stage. The open source platform SciLab is chosen because of high computational power, statistical and graphic functions useful for results interpretation and the possibility to benefit of the support and the frequent update provided by SciLab Consortium.

VirtES basic rule for code implementation is to develop a single SciLab function for each model process. Each function could be organized hierarchically for a better code design. The process interaction diagram represents also the main flow - diagram of the SciLab code. If the studied enterprise fits with an existing reference model the function already developed for previous studies can be reused. This feature contributes to save resources and time during the model development. The SciLab model is able to simulate the behavior of the enterprise and gives results in term of the chosen macro KPI (Key Performance Indicators).

\subsection{The Third Stage}

At the third stage sub - models of specific process or sub - system are developed. The most appropriate mathematical formalism and software tools could be used to achieve the expected results. In fact the possibility to interface the sub - models with the main SciLab model is demanded to the open - source platform potential.

\section{The Case - Study}

VirtES methodology was full developed and firstly applied to ceramic tiles enterprises of the Emilian ceramic cluster. A reference FDM model is developed and coded in SciLab. In the reference model are defined the significant features for the characterization of a tile enterprise and the macro KPI to evaluate the performances.

The models developed by VirtES allowed to quantify for a generic tile enterprise the potential economic benefits related to different BPR actions.

\subsection{Model Implementation}

The reference model is developed starting from studied enterprises and validated with the evidence found in literature. The description of the manufacture system provided by Andres is confirmed [7]. The model includes also the order process in order to describe the entire enterprise behavior from order collection to costumer satisfaction. The amount of sold tiles (named "SPE") and the average stock level (named "MAG") are chosen as macro KPI, a representation is given in figure 3 . The complete tile enterprise reference model is provided in figure 4. 


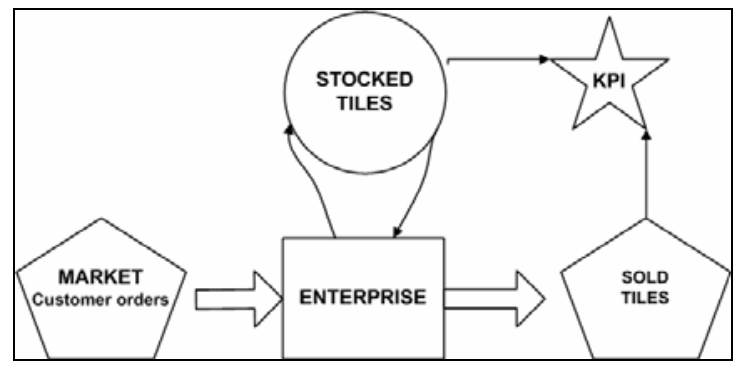

Fig. 3. Tile enterprise KPI

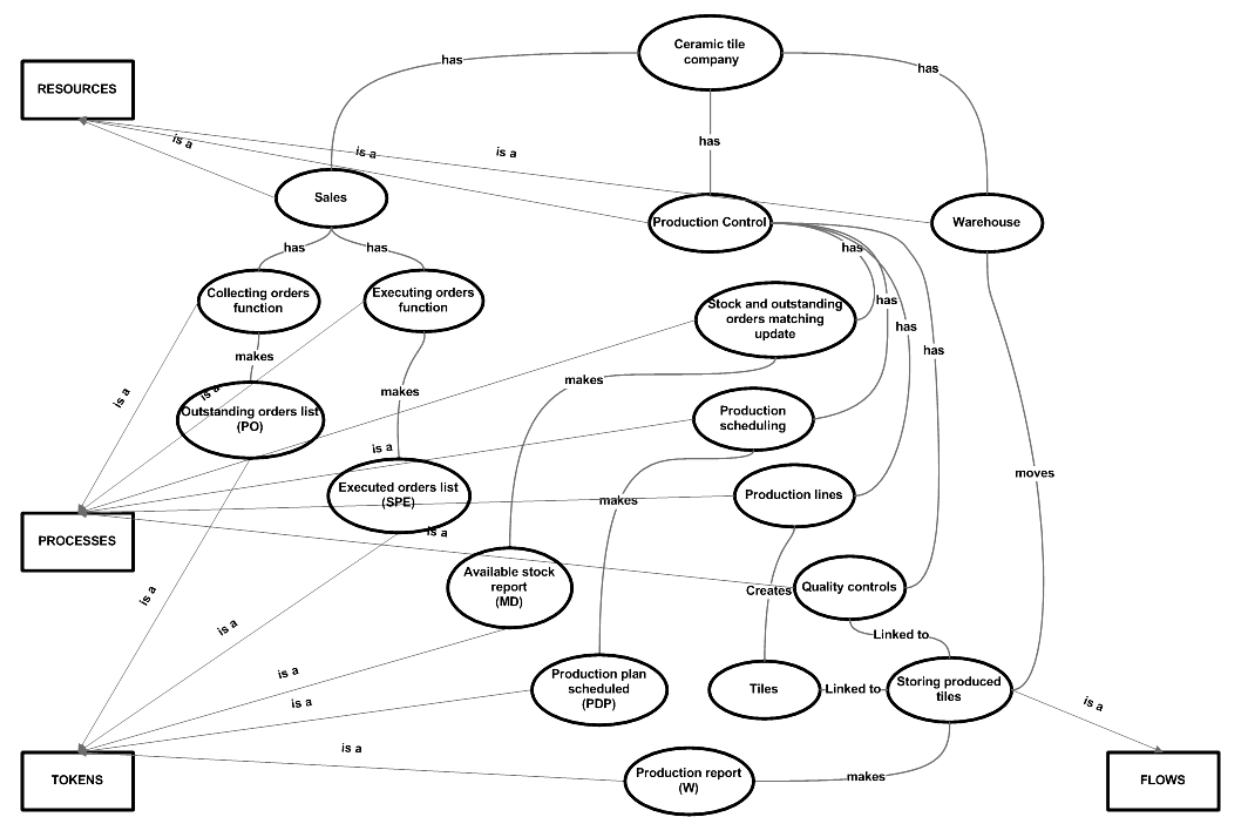

Fig. 4. Tile enterprise reference FDM model

The simulation model is provided and every process is coded in a single SciLab function. The inputs for the FDM model are customer orders, the SciLab simulation model could be feed by real orders from historical data or by a random function that generates orders according to the imposed rules.

The simulation model is set according to the characteristics of the studied enterprise and validated. After validation process the model is used to investigate the effectiveness of the considered BPR actions:

- IT: order process and IT (Information Technology) re - engineering;

- TQ: manufacturing system improvement toward TQ (Total Quality);

- FO: sales forecast optimization (FO).

In the first case the enterprise performances are simulated in the hypothesis of a complete reliability of orders data base information. In the second case the hypothesis of 
the absolute absence of color tone variation in final products is taken, [8]. In the third case the system behavior is simulated under the hypothesis of total according with sales forecast and real market orders. Simulating the enterprise performance in these extreme condition is useful to evaluate the potentiality of each BPR action.

An integration with the production line sub-model, developed with AutoMod, is provided to evaluate the effect of BPR actions at production line level [9].

\subsection{Results}

To enable an economic analysis, a simple function is proposed, termed the "Earning function" (1). Maximizing the proposed function means that the performance of the company, as defined in the model, is optimized in terms of enterprise profit. The simulation results are reported in table 1. referring to a period of one year.

$$
\mathrm{f}(\mathrm{SPE}, \mathrm{MAG})=\mathrm{M} * \mathrm{SPE}-\mathrm{C}_{\mathrm{m}} * \mathrm{MAG} \text {. }
$$

- $\mathrm{M}$ is the spread between the average sale price and the average production cost for $1 \mathrm{~m}^{2}$ of tiles;

- $\mathrm{C}_{\mathrm{m}}$ is the average stocking cost for $1 \mathrm{~m}^{2}$ of tiles for 1 year.

High-level simulation results point out the expected improvement in the enterprise profits related to the adoption of any considered BPR action; the results are provided in table 1.

Table 1. Simulation results

\begin{tabular}{l|r}
\hline \multicolumn{1}{c|}{ BPR actions } & Enterprise profits $(€)$ \\
\hline None, present state & 961.211 \\
IT & 999.871 \\
TQ & 993.151 \\
FO & 965.883 \\
IT + TQ & 1.095 .525 \\
\hline IT + FO & 1.010 .605 \\
\hline
\end{tabular}

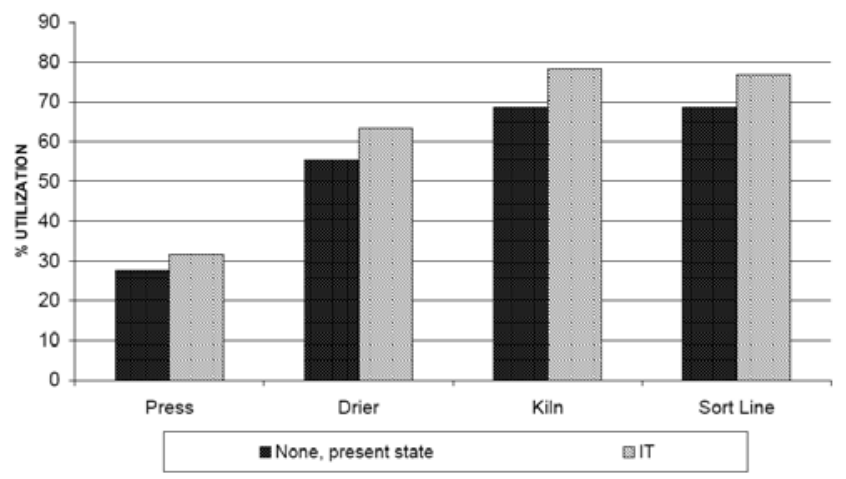

Fig. 5. Sub-model results in term of machineries utilization 
The results of the low-level AutoMod model show the improvement in machineries utilization; the results are shown in figure 5.

The most promising single BPR action is IT re - engineering. Also IT re engineering positive impact on machinery utilization is quantified tanks to the detailed sub - model.

\section{Conclusion}

The VirtES methodology prevents from developing a very detailed model for the whole enterprise and allows to create and integrate detailed modes for specific sub systems. The proposed approach requires low resources and matches the instances of SMEs. The implementation of the present approach provides an useful tool for the enterprise to support management in decision-making, investment planning and improvement strategy defining. The results achieved, applying VirtES to the Italian ceramic industry [10] encourage the adoption to other industrial field.

\section{References}

1. Al-Aoma, R.: Product-mix analysis with discrete event simulation. In: 2000 Winter Simulation Conference, pp. 10385-10392. WSC Press, Orlando (2000)

2. Vergidis, K., Turner, C.J., Tiwari, A.: Business process perspectives: Theoretical developments vs. real-world practice. Int. J. Production Economics 114, 91-104 (2008)

3. Shannon, R., Long, S., Buckles, B.: Operations research methodologies in industrial engineering. AIIE Transactions 12, 364-367 (1980)

4. Ryan, J., Heavey, C.: Process modelling for simulation. Computer in Industry 57, 437-450 (2006)

5. O'Kane, J., Papadoukakis, A., Hunte, D.: Simulation usage in SMEs. Journal of Small Business and Enterprise Development 14, 512-552 (2007)

6. Yu, B., Harding, J.A., Popplewell, K.: A reusable enterprise model. International Journal of Operations \& Production Management 20, 50-69 (2001)

7. Andrés, C., Albarracin, J.M., Torino, G., Vicens, E., Garcia-Sabater, J.P.: Group tschnology in a hybrid flowshop environment: A case study. European Journal of Operational Research 167, 181-272 (2005)

8. Erginel, N., Dogan, B., Ay, N.: The statistical analysis of coloring problems faced in ceramic floor tile industry. In: 8th Conference and Exhibition of the European Ceramic Society, pp. 1693-1696. ECS Press, Istanbul (2003)

9. Davoli, G., Gallo, S.A., Melloni, R.: Analysis of industrial processes based on integration of different simulation tools. In: 10th MITIP International Conference, pp. 38-44. MITIP Press, Prague (2008)

10. Davoli, G., Gallo, S.A., Melloni, R.: Analysing the ceramic sector using processes and simulation models. Ceramic World Review 80, 116-119 (2009) 